\title{
Adsorption and Interlayer Diffusion Controlled Growth and Unique Surface Patterned Growth of Polyelectrolyte Multilayers
}

\author{
Jing Yu, Brooke M. Meharg, Ilsoon Lee*
}

\begin{abstract}
In this work, extremely high molecular weight $\left(M_{w}\right)$ poly(allylamine hydrochloride) (PAH, 900K g/mol) and poly(acrylic acid) (PAA, $225 \mathrm{~K} \mathrm{~g} / \mathrm{mol}$ ) were selected to amplify the difference in the growth of multilayers in comparison with low $M_{w}$ PAA $(15 \mathrm{~K} \mathrm{~g} / \mathrm{mol})$ and PAH $(15 \mathrm{~K}$ $\mathrm{g} / \mathrm{mol}$ ). By varying the $\mathrm{pH}$ conditions, the PAH/PAA multilayers were fabricated via the layerby-layer (LbL) assembly in both linear and exponential growth regimes. In the linear growth regime with interlayer diffusion suppressed, high $M_{w}$ polyelectrolytes with low charge density could slow down the adsorption step, leading to the decrease of thickness compared to low $M_{w}$ polyelectrolytes when the deposition time was limited. However, the effect of $M_{w}$ could be reversed by increasing the deposition time, for the adsorption of low $M_{w}$ polyelectrolyte reached equilibrium, while the adsorption of high $M_{w}$ polyelectrolyte continued. The larger coil size of high $M_{w}$ polyelectrolyte could enable the surpassing of multilayer thickness compared with low $M_{w}$ polyelectrolyte. In the exponential growth regime, besides the slow adsorption, the application of high $M_{w}$ polyelectrolytes further suppressed the interlayer diffusion, leading to the decrease of multilayer thickness regardless of deposition time. We further studied the effects of deposition time, $M_{w}$ of polyelectrolytes, and the number of bilayers on the surface morphology in the exponential growth regime. Surface roughness significantly increased with the application of
\end{abstract}


high $M_{w}$ PAA and the increase in deposition time. For the first time, the unique LbL film growth patterns of islet, ring and cantaloupe skin-like structures that consecutively formed on the surface of polyelectrolyte multilayers were reported by tuning the above parameters.

Key words: polyelectrolyte multilayers, film growth, adsorption, interlayer diffusion, surface morphology

\section{Introduction}

Polyelectrolyte multilayers (PEMs) are formed by the alternate deposition of polyanions and polycations onto the solid surfaces via layer-by-layer (LbL) assembly.[1] These films have received extensive attention during the past two decades due to their potential applications in the fields of fabricating functional thin films and coatings.[1-3] LbL assembled films have been engineered for drug delivery systems[4-6], anti-bacterial surfaces[7, 8], substrates for cell adhesion and proliferation $[9,10]$, gas barrier[11, 12], gas separation[13], membrane filtration[14, 15], anti-reflection coatings[16], electrical conductive devices[17], etc.

LbL assembly is considered as a versatile technique as it is highly tunable and can control the incorporation of functional materials, film thickness, composition and polymer distribution, according to different applications. There are two possible steps which may dominate the growth

of the multilayers as shown in Schematic 1(a). One is adsorption, which is the step of polyelectrolyte molecules moving from the solution to the film surface. The other is the interlayer diffusion, indicating the movement of polyelectrolyte molecules from the surface into the film. So far, two types of multilayer growth behaviors have been reported based on the two steps. (1) Linear growth, where the thickness or mass of the film increases linearly with the 
number of bilayers. Linear growth is adsorption controlled with the polyelectrolytes in the solution only interacting with the oppositely charged polyelectrolytes on the film surface. [18, 19] (2) Exponential growth, where the films grow exponentially with the number of bilayers. The exponential growth behavior is based on the interlayer diffusion within the PEMs, which always happens in weak polyelectrolyte systems if assembled with partially charged polyelectrolytes.[20-23] Recent study showed that the charge mismatch between poly(allylamine hydrochloride) $\mathrm{PAH}$ and poly(acrylic acid) PAA controlled by the $\mathrm{pH}$ conditions of the polyelectrolyte solutions was associated with interlayer diffusion, and in turn was able to control the growth behavior of the multilayers.[24]

Besides $\mathrm{pH}$ of polyelectrolyte solutions, the deposition time also affects the film growth significantly as the deposition process of polyelectrolyte is time-dependent. [25-28] Normally it would be expected that the increase of deposition time could lead to a thicker film. However, Barrett et al.[27] assembled the multilayers using PAH and a polyanion containing an azobenzene chromophore (P-Azo) and found a significant increase of the film thickness when the deposition time was less than $5 \mathrm{~s}$. Similar results were obtained by from poly (ethylene imine) (PEI)/PAA/PEI/ montmorillonite (MMT) clay quadlayer system. [26] It has also been discovered that the PEI/PAA films grew linearly with deposition time of $1 \mathrm{~s}$ and exponentially with longer deposition time.[28] For a short deposition time, due to the time limit for interlayer diffusion, the film growth is adsorption controlled, leading to a linear growth behavior.

Another important parameter which affects the growth behavior of the multilayers is the molecular weight of polyelectrolyte. For linear growth behavior, the film thickness increased with the increase of molecular weight of polyelectrolytes because of the more coiled polyelectrolyte chains with high molecular weight.[19, 29] However, with the existence of 
interlayer diffusion, the film thickness increased with the decrease of the molecular weight of polyelectrolytes, indicating a fast diffusion of low molecular weight polyelectrolytes.[30, 31] For hyaluronan (HA)/chitosan (CHI) multilayers[32], in which $\mathrm{CHI}$ is the diffusing polyelectrolyte and HA is the non-diffusing species, the film thickness increased with the molecular weight increase of both polyelectrolytes. In the case of HA/ poly(L-lysine) (PLL) multilayers[33], the low molecular weight PLL can diffuse into the entire film during the deposition step; whereas the diffusion of the high molecular weight PLL was restricted to the upper part of the film. However, the film thickness still increased with the increase of molecular weight of PLL, while the increase of molecular weight of HA led to a decrease of the film thickness. Helm et al.[34, 35] found that the molecular weight of polyelectrolytes shifted the growth regime of poly(diallyldimethylammonium) (PDADMAC)/ poly(styrenesulfonate) (PSS) multilayers. In general, it is difficult to predict the effect of molecular weight of polyelectrolytes on the growth behavior of multilayers. The previous studies only considered the molecular weight of polyelectrolytes as an independent variable for influencing the film growth. [19, 29, 31-33] However, we believe the effect of molecular weight is intertwined with other parameters including the deposition time and the charge density of the polyelectrolytes.

Besides film growth, surface topography of multilayers also changes with the number of bilayers, molecular weight of polyelectrolytes and the $\mathrm{pH}$ of polyelectrolyte solutions. For CHI/HA multilayers, the small islets showed up on the surface after the deposition of the first bilayer. The individual islets coalesced into larger vermiculate features with the increase of the number of bilayers. $[30,32]$ In addition, the transition from small islets to vermiculate patterns depends on the molecular weight of the polyelectrolytes. The low molecular weight polyelectrolytes could facilitate the generation of larger wormlike structures than the high 
molecular weight polyelectrolytes.[32] For PAH/PAA mutilayers, the surface roughness increased from 5.5 to $6.7 \mathrm{~nm}$ with the increase of molecular weight of PAA from 7000 to 49000 g/mol. Shen et al. [36] prepared PEI/PAA multilayers with PEI at high pH and PAA at low $\mathrm{pH}$. The special deposition conditions facilitated the interlayer diffusion of PEI, leading to the exponential growth of the films and the formation of micro/nano hierarchical structures. However, there is no study about the effect of the deposition time on the surface topography of the multilayers.

PEMs show different growth behavior depending on the type of polyelectrolytes, deposition time as well as the molecular weight. In order to design LbL multilayers successively and extend their applications, it is necessary to understand the build-up of multilayers. PAH and PAA are among the most widely used polyelectrolytes used for a variety of applications. There are however, a number of parameters which can affect the deposition of these films but haven't been studied in detail thus far. In the present work, the growth behavior of PAH/PAA multilayers was controlled in both linear and exponential regimes by fabricating the multilayers under different pH conditions. Two distinct molecular weights for PAA $(15,000$ and 225,000 g/mol) and PAH $(15,000$ and $900,000 \mathrm{~g} / \mathrm{mol})$ were chosen to study the molecular weight effect on the film growth in detail. The application of polyelectrolytes with distinct molecular weights amplified the differences of chain mobility, and therefore enabled the observation of very detailed changes during the deposition steps. In this work, we demonstrated that the effect of molecular weight of polyelectrolytes on the film growth is dependent on the deposition time and charge density. We also proved that high molecular weight polyelectrolytes could not only suppress the interlayer diffusion but also slow down the adsorption step significantly. In addition, by tuning the molecular weight of polyelectrolytes, deposition time and number of bilayers, we were able to 
obtain unique surface topographies of PAH/PAA multilayers ranging from nano- to micro-scale. It is expected that the results reported herein will be of interest for a better understanding of the build-up of weak polyelectrolyte multilayers.

\section{Experimental Section}

\section{Materials}

Poly(acrylic acid) $\left(\mathrm{PAA}_{\mathrm{L}}, M_{w}=15,000 \mathrm{~g} / \mathrm{mol}, 35 \%\right.$ aqueous solution, and $\mathrm{PAA}_{\mathrm{H}}$, $M_{w}=225,000 \mathrm{~g} / \mathrm{mol}, 20 \%$ aqueous solution) was purchase from Sigma Aldrich and Polyscience, respectively. Both poly(allylamine hydrochloride) $\left(\mathrm{PAH}_{\mathrm{L}}, M_{w}=15,000 \mathrm{~g} / \mathrm{mol}\right.$ and $\mathrm{PAH}_{\mathrm{H}}$, $M_{w}=900,000 \mathrm{~g} / \mathrm{mol}$ ) were purchased from Sigma-Aldrich. All aqueous solutions were prepared using $18 \mathrm{M} \Omega$ Millipore water, generating by a Barnstead Nanopure Diamond-UV purification unit equipped with a UV source and a final $0.2 \mu \mathrm{m}$ filter. Glass slide, silicon wafer, polycarbonate, and polystyrene were used as substrates for LbL assembly and cleaned extensively prior to the deposition.

\section{Layer-by-Layer Assembly}

All LbL films were assembled with a programmable Carl-Zeiss slide-stainer. Before depositing the multilayers, the substrate was cleaned by a plasma cleaner (Harrick Scientific Corporation, Broading Ossining, NY). The films were subjected to oxygen plasma for $20 \mathrm{~min}$, producing hydrophilic moieties and negative charges on the surface. After the oxygen plasma treatment, polyelectrolyte solutions were prepared $\left(10^{-2} \mathrm{M}\right.$ based on repeat unit of the polymer) with $18 \mathrm{M} \Omega$ Millipore water and were $\mathrm{pH}$-adjusted with $0.1 \mathrm{M} \mathrm{HCl}$ or $\mathrm{NaOH}$. The substrate was first dipped into PAH solution (without adjusting the $\mathrm{pH}$ ) for $20 \mathrm{~min}$ to form a precursor layer, follow by three washing steps. Then, substrate was introduced in the aqueous solution of PAA adjusted to a desired $\mathrm{pH}$ for certain amount of deposition time, followed by three washing steps 
with $\mathrm{pH}$ adjusted DI water for sufficient amount of time. Subsequently, the substrate was immersed in the PAH aqueous solution adjusted to a desired $\mathrm{pH}$ with the same deposition time as PAA, and washed again three times with $\mathrm{pH}$ adjusted DI water. The $\mathrm{pH}$ of DI water for three washing steps was kept the same as the $\mathrm{pH}$ of the previous polyelectrolyte solution. The deposition process was repeated until the desired number of bilayers was obtained. Different deposition time has been tried in this study including 10s, 1 min, 5min, 10min, $15 \mathrm{~min}$ and $30 \mathrm{~min}$.

\section{Film Characterization}

The thickness and surface roughness of the PEM films were measured in the dry state with a Dektak surface profiler. The surface topography and the roughness were measured by an Asylum Research Cypher ${ }^{\mathrm{TM}}$ Atomic Force Microscope/Scanning Probe Microscope (AFM/SPM) (Santa Barbara, CA) performed using tapping mode. A JEOL 6610LV Scanning Electron Microscopy (SEM) was used to observe morphology of the surface. All specimens were coated with gold before examination.

\section{Results and Discussion}

Effect of Deposition Time on the Thickness of PAH/PAA Multilayers. The thickness values of (PAH8.5/PAA3.5) 20.5 films are presented in Figure 1 as a function of deposition time. The polyelectrolyte multilayers were constructed by the alternate deposition of $\mathrm{PAH}$ at $\mathrm{pH}=8.5$ and PAA at $\mathrm{pH}=3.5$. We applied FTIR to characterize the degree of ionization of polyelectrolytes in the aqueous solutions with different molecular weight (data not shown).[21] It was found that when $\mathrm{pH}=3.5$, the degree of ionization of low molecular weight PAA was $20 \%$; while the degree of ionization of high molecular weight PAA was $18 \%$. Moreover, the degree of ionization of $\mathrm{PAH}$ with low and high molecular weight at $\mathrm{pH}=8.5$ was $58 \%$ and $61 \%$, 
respectively. Therefore, the molecular weight did not affect the degree of ionization of polyelectrolytes. According to the previous studies[22, 24, 31], the interlayer diffusion of both PAA and PAH led to an exponential growth of the multilayers. When weak polyelectrolytes are partially charged during LbL assembly, the mobility and swelling of the multilayers facilitate the interlayer diffusion.[23, 37] As shown in Figure 1, the thickness of (PAH8.5/PAA3.5) 20.5 films increased with the deposition time, which varied from $10 \mathrm{~s}$ to $15 \mathrm{~min}$, for the adsorption of polyelectrolytes, interlayer diffusion and the reconstruction of the multilayers always take time to reach the equilibrium.[23, 37] With shorter deposition time, the interlayer diffusion was highly limited, leading to a decrease of film thickness.

\section{Effect of Molecular Weight of Polyelectrolytes on the Film Growth of PAH/PAA}

Multilayers. In order to study the molecular weight effect thoroughly, we fabricated PAH/PAA multilayers using PAA with molecular weight of $15,000 \mathrm{~g} / \mathrm{mol}\left(\mathrm{PAA}_{\mathrm{L}}\right)$ and $225,000 \mathrm{~g} / \mathrm{mol}$ $\left(\mathrm{PAA}_{\mathrm{H}}\right)$ and PAH with molecular weight of 15,000 g/mol $\left(\mathrm{PAH}_{\mathrm{L}}\right)$ and 900,000 g/mol $\left(\mathrm{PAH}_{\mathrm{H}}\right)$. Figure 1 also illustrates the effect of molecular weight of polyelectrolytes on the thickness of (PAH8.5/PAA3.5) 20.5. It is obvious that the increase of molecular weight of polyelectrolytes led to a decrease of film thickness. However, with a less increase in molecular weight of PAA, there was a more significant decrease in the film thickness, as compared to PAH. When the deposition time increased from $10 \mathrm{~min}$ to $15 \mathrm{~min}$, for low molecular weight PAA systems, the increase of thickness became very limited; whereas for multilayers with high molecular weight PAA, the thickness of the multilayers still increased significantly. This indicates that the increase of molecular weight of PAA slowed down the deposition process. Even though the increase of molecular weight of PAH led to a decrease of film thickness, it did not influence the thickness as much as the molecular weight of PAA did. In addition, when the deposition time was shortened 
to 10 s, the molecular weight of PAH almost did not affect the film thickness, while the increase of molecular weight of PAA led to a decrease of film thickness from around $466 \mathrm{~nm}$ to around $245 \mathrm{~nm}$. The interlayer diffusion was highly suppressed when the deposition time was 10s, regardless of the molecular weight of polyelectrolytes. Thus, the suppression of film growth indicates that the low chain mobility of high molecular weight PAA with low the degree of ionization at $\mathrm{pH}=3.5$ may lead to a slower adsorption step; whereas the adsorption for PAH did not vary by the molecular weight probably due to its relatively high degree of ionization at $\mathrm{pH}=$ 8.5 .

In order to confirm this idea, we further assembled the multilayers at $\mathrm{pH}$ conditions of PAH10/PAA10 (PAH with low charge density/ PAA with high charge density) and PAH3.5/PAA3.5 (PAH with high charge density/ PAA with low charge density), respectively. The molecular weight of PAA and the deposition time were also varied. The thickness data are summarized in Table 1. The simplified scenarios for the deposition step of PAA under different LbL assembly conditions are illustrated in Schematic 1 (b) - (d). Based on the previous study[24], when assembled at $\mathrm{pH}$ conditions of PAH10/PAA10 and PAH3.5/PAA3.5, the multilayers followed the linear growth behavior due to the significant difference in charge density between PAA and PAH, which limited the interlayer diffusion. According to the thickness data in Table 1, when the multilayers fabricated under PAH10/PAA10, the increase of molecular weight of PAA led to an increase of the film thickness, which is consistent with previous studies[19, 29]. As presented in Schematic 1 (b), if the degree of ionization of PAA is very high, the adsorption is fast due to the strong electrostatic interaction between the multilayer surface and the molecular chain. With suppressed interlayer diffusion, the film thickness increased with the molecular weight of PAA because the polymer chains of high molecular weight PAA are more coiled than 
those of low molecular weight PAA. In the case of PAH3.5/PAA3.5, the interlayer diffusion is also suppressed. However, the film thickness decreased with the increase of molecular weight of PAA when the deposition time was $5 \mathrm{~min}$. By increasing deposition time to $30 \mathrm{~min}$, the thickness of the film fabricated with high molecular weight PAA further increased and surpassed the thickness of the film fabricated with low molecular weight PAA. When the degree of ionization of PAA is very low, the electrostatic interaction between the multilayer surface and polymer chains is weakened as shown in Schematic 1 (c). The increase of molecular weight of PAA further slowed down the adsorption step due to its poor chain mobility. Therefore, the multilayers with high molecular weight PAA were built up more slowly, leading to an initial decrease of thickness compared to low molecular weight PAA. With the increase of deposition time, the deposition of low molecular weight PAA reached the equilibrium while the deposition of high molecular weight PAA continued. Eventually, the film thickness with high molecular weight PAA exceeded the film thickness with low molecular weight PAA due to the larger coil size of high molecular weight PAA. Schematic 1 (d) shows the case with interlayer diffusion taking place when the degree of ionization of PAA was relatively low. For low molecular weight PAA, the adsorption was fast, therefore the interlayer diffusion was the control step for film growth. Meanwhile, high molecular weight PAA suppressed the adsorption leading to the decrease of film thickness when the deposition time was $10 \mathrm{~s}$. With the increase of deposition time, the interlayer diffusion was also suppressed by the high molecular weight PAA. Due to the time scale of the experiments, we did not try to figure out the deposition time required to reach the deposition equilibrium for high molecular weight PAA. Moreover, similar results were observed for PAH under the condition of PAH10/PAA10, when PAH was weakly charged. As shown in Table 1, with the application of high molecular weight PAH, the multilayer thickness 
increased when the deposition time increased from 5 to $15 \mathrm{~min}$, indicating a slow adsorption step. Therefore, it can be concluded that not only interlayer diffusion can affect the growth behavior of the PEMs, the adsorption step is also critical especially for high molecular weight polyelectrolytes with low degree of ionization. 

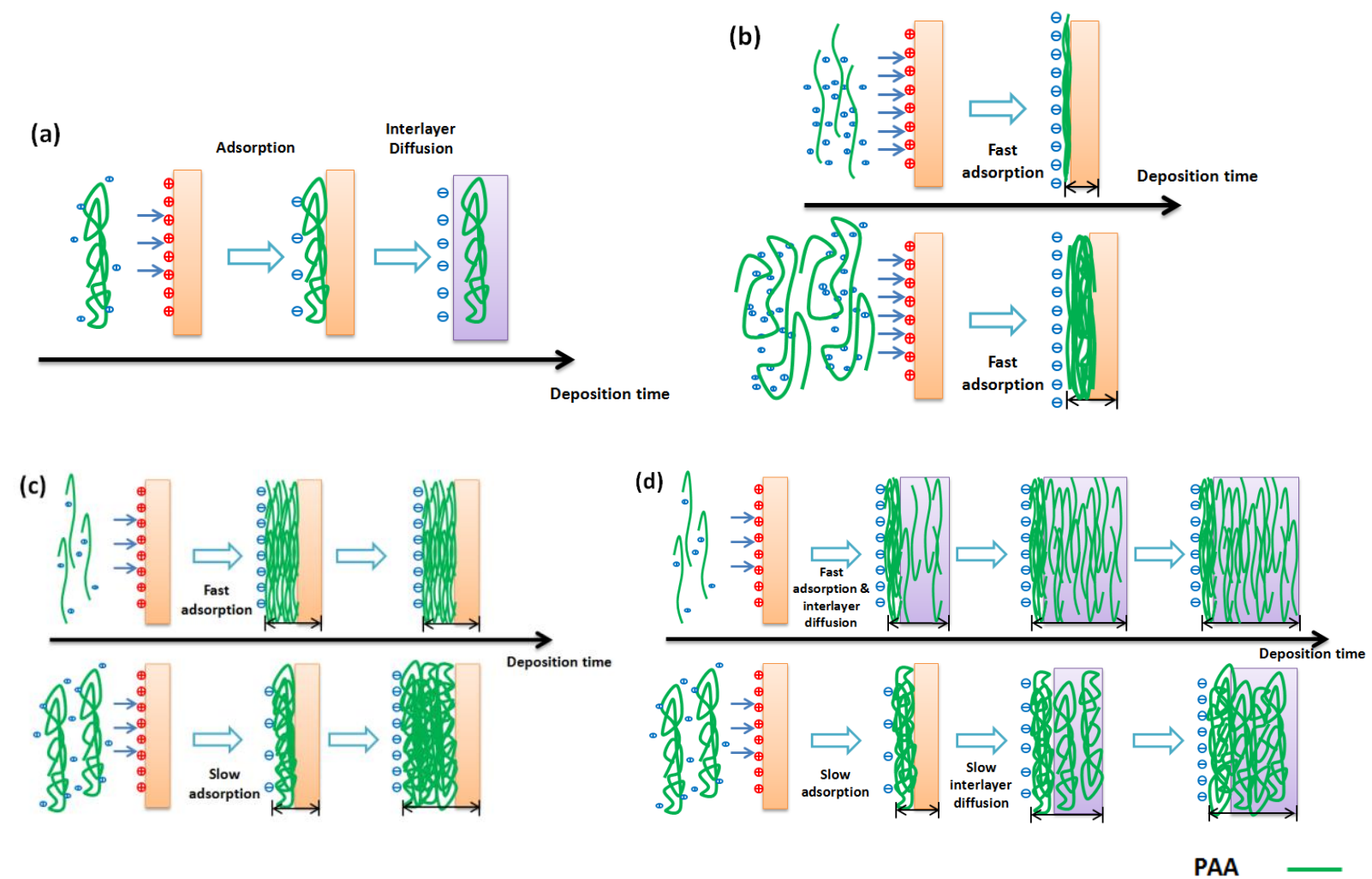

Schematic 1. Representations of (a) the definition of adsorption and interlayer diffusion during the deposition process and (b) - (d) the deposition process of PAA with different molecular weight under the condition of PAH10/PAA10, PAH3.5/PAA3.5, and PAH8.5/PAA3.5, respectively. (The charge density or zeta potential on the multilayer surface varied by changing the $\mathrm{pH}$ conditions. However, the deposition of the low and high molecular weight PAA were only compared under the same deposition conditions.) 


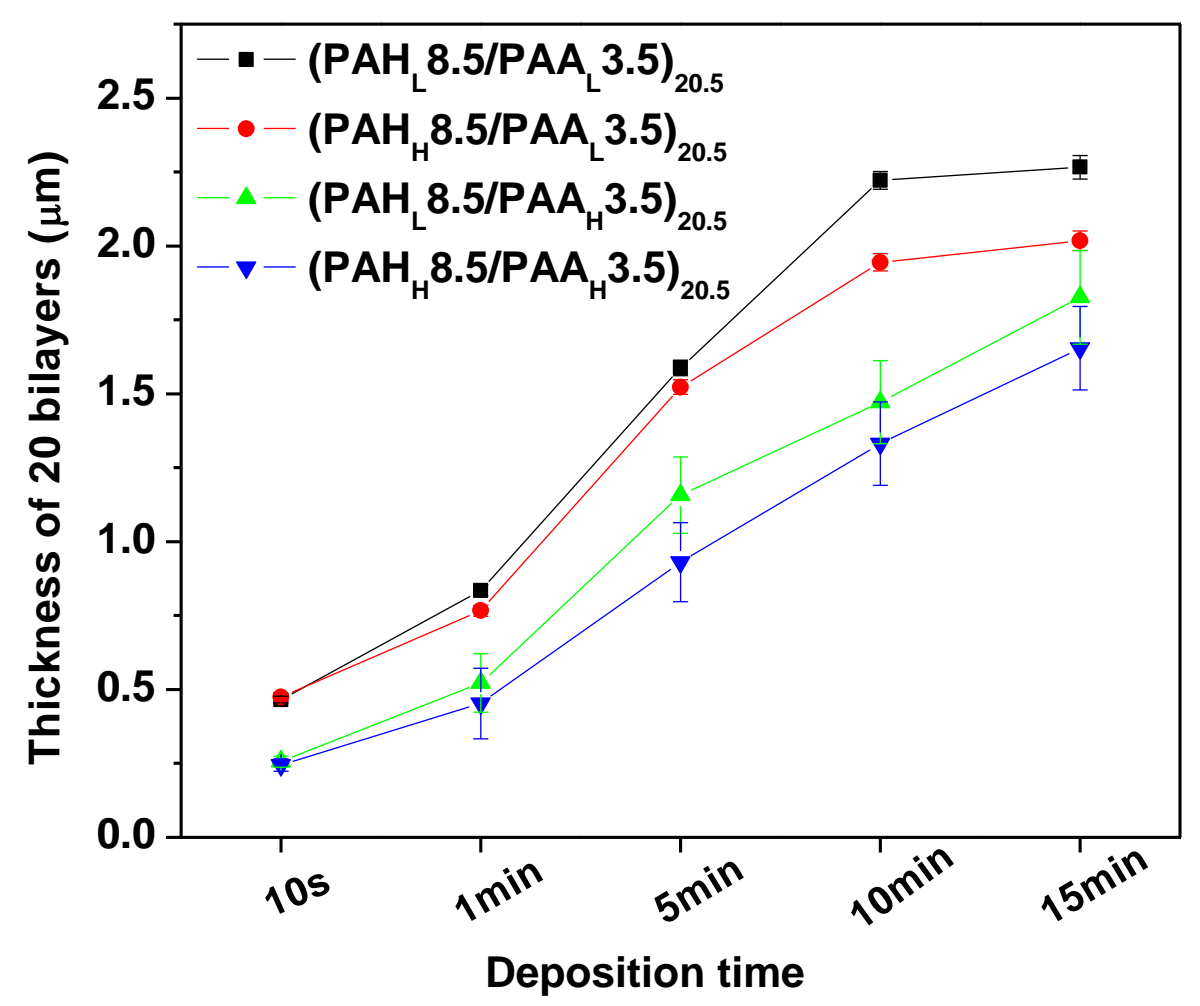

Figure 1. The effect of deposition time and molecular weight of polyelectrolytes on the thickness of (PAH8.5/PAA3.5) 20.5 films. These thickness data were acquired with profiler.

Table 1. Thickness of (PAH/PAA $)_{20.5}$ multilayers fabricated under different $\mathrm{pH}$ conditions

\begin{tabular}{|c|c|c|}
\hline Samples & Deposition time (min) & Thickness $(\mu \mathrm{m})$ \\
\hline$\left(\mathrm{PAH}_{\mathrm{H}} 8.5 / \mathrm{PAA}_{\mathrm{L}} 3.5\right)_{20.5}$ & 5 & $1.523 \pm 0.026$ \\
\hline$\left(\mathrm{PAH}_{\mathrm{H}} 8.5 / \mathrm{PAA}_{\mathrm{H}} 3.5\right)_{20.5}$ & 5 & $0.931 \pm 0.014$ \\
\hline$\left(\mathrm{PAH}_{\mathrm{H}} 10 / \mathrm{PAA}_{\mathrm{L}} 10\right)_{20.5}$ & 5 & $0.017 \pm 0.005$ \\
\hline$\left(\mathrm{PAH}_{\mathrm{H}} 10 / \mathrm{PAA}_{\mathrm{L}} 10\right)_{20.5}$ & 30 & $0.060 \pm 0.006$ \\
\hline$\left(\mathrm{PAH}_{\mathrm{H}} 10 / \mathrm{PAA}_{\mathrm{H}} 10\right)_{20.5}$ & 5 & $0.065 \pm 0.009$ \\
\hline$\left(\mathrm{PAH}_{\mathrm{H}} 3.5 / \mathrm{PAA}_{\mathrm{L}} 3.5\right)_{20.5}$ & 5 & $0.118 \pm 0.005$ \\
\hline$\left(\mathrm{PAH}_{\mathrm{H}} 3.5 / \mathrm{PAA}_{\mathrm{L}} 3.5\right)_{20.5}$ & 30 & $0.123 \pm 0.006$ \\
\hline$\left(\mathrm{PAH}_{\mathrm{H}} 3.5 / \mathrm{PAA}_{\mathrm{H}} 3.5\right)_{20.5}$ & 5 & $0.108 \pm 0.008$ \\
\hline$\left(\mathrm{PAH}_{\mathrm{H}} 3.5 / \mathrm{PAA}_{\mathrm{H}} 3.5\right)_{20.5}$ & 30 & $0.129 \pm 0.003$ \\
\hline
\end{tabular}




\section{Effect of Deposition Time on the Surface Topography of PAH/PAA Multilayers. The}

SEM images of the surface topography of (PAH8.5/PAA3.5) 20.5 films are shown in Figure 2. For the multilayers fabricated with low molecular weight of PAA, the surfaces were relatively flat and featureless under the SEM. However, the detailed structure was not very evident from the SEM images. We further applied AFM to characterize the surface topography. Figure 3 and 4 consist of the AFM 3D images for $\left(\mathrm{PAH}_{\mathrm{L}} 8.5 / \mathrm{PAA}_{\mathrm{L}} 3.5\right)_{20.5}$ and $\left(\mathrm{PAH}_{\mathrm{H}} 8.5 / \mathrm{PAA}_{\mathrm{L}} 3.5\right)_{20.5}$, respectively. As shown in Figure 3(a) and 4(a), the surface contained a lot of small islets for short deposition time. With the increase of deposition time, the surface became smoother with larger peak and valley structures. For long deposition time, the polymer chains have enough time to relax and reconstruct on the surface as well as inside the multilayers, leading to a smoother surface; while for short deposition time, the polymer chains have limited time to settle down on the surface, leading to the formation of the small islets. A progressive roughness increase is observed in Figure 2 with increasing deposition time for high molecular weight PAA systems. When the deposition time was $10 \mathrm{~s}$, the surface was relatively flat. Thus, the AFM 3D images for four systems with the deposition time of $10 \mathrm{~s}$ are shown in Figure 5 for comparison. As shown in Figure 5 (c) and (d), the surface consisted of small sharp islets. This island like structure was significantly enhanced when the deposition time increased to $5 \mathrm{~min}$ in Figure 2. When the deposition time further increased to 10 or $15 \mathrm{~min}$, a micro-scale network structure was formed on the surface, which is very similar to the skin structure of cantaloupe. The surface RMS roughness data of all four multilayer systems are listed in Table 2. In general, the RMS roughness increased with the deposition time. 


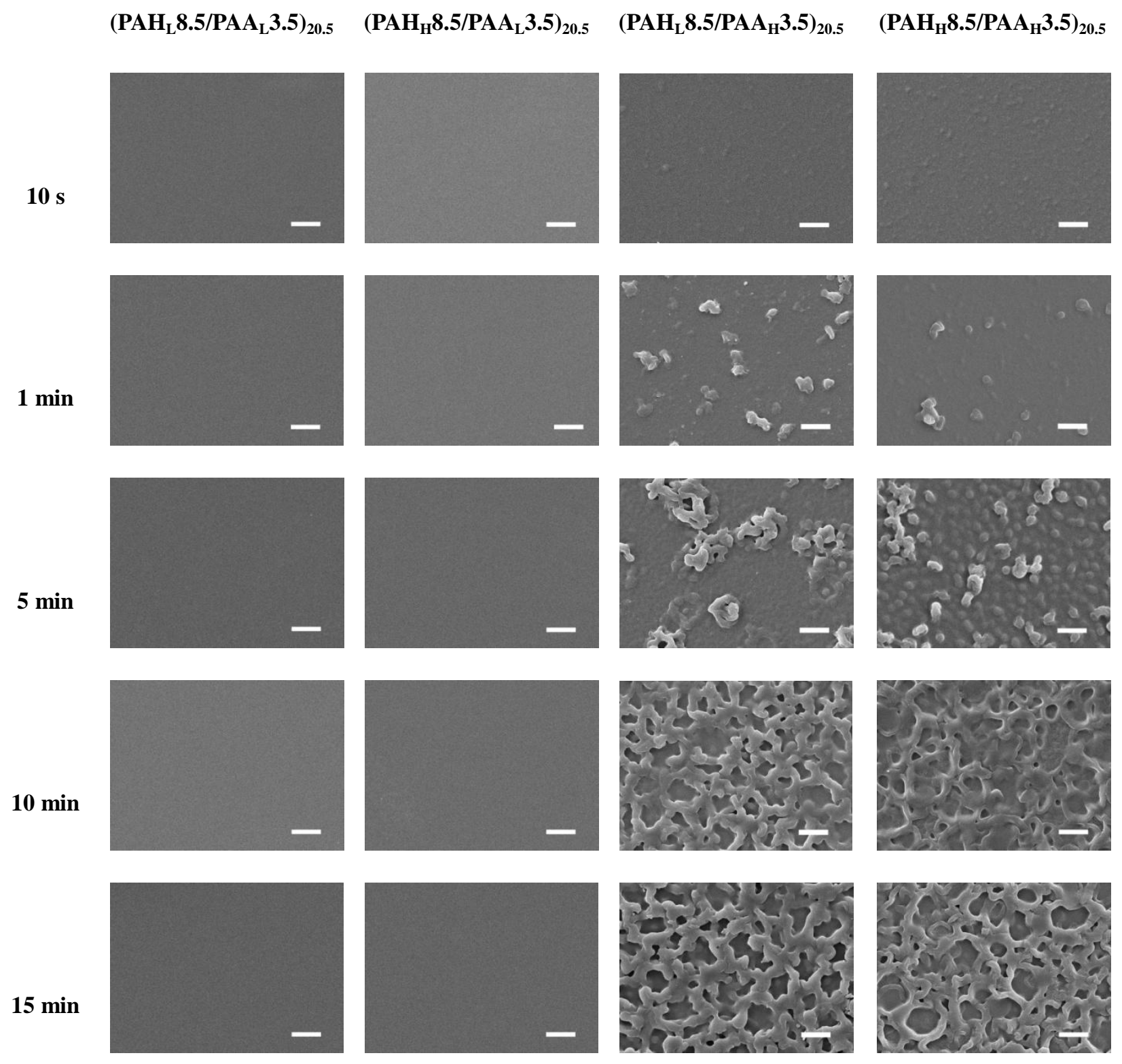

Figure 2. SEM images of surface morphology of (PAH8.5/PAA3.5) ${ }_{20.5}$ films. (The scale bar is $5 \mu \mathrm{m}$ for all images.)

\section{Effect of Molecular Weight of Polyelectrolytes on the Surface Topography of}

PAH/PAA Multilayers. It is obvious from Figure 2 that the application of high molecular weight PAA enabled the formation of certain patterns on the multilayer surfaces and increased the surface roughness significantly, when the deposition time increased to more than $10 \mathrm{~s}$. Even when the deposition time was $10 \mathrm{~s}$, comparing to the surface of the multilayers built up with low 
molecular weight PAA (Figure 5 (a) and (b)), the height of the islets on the surface increased significantly with high molecular weight PAA (Figure 5 (c) and (d)), leading to the increase of RMS roughness. In all, the application of high molecular weight PAA led to the increase of surface roughness, which is also consistent with the RMS roughness listed in Table 2.

Table 2. Surface roughness of (PAH8.5/PAA3.5) ${ }_{20.5}$ films

\begin{tabular}{|c|c|c|}
\hline Samples & Deposition Time & Roughness (nm) \\
\hline \multirow{5}{*}{$\left(\mathrm{PAH}_{\mathrm{L}} 8.5 / \mathrm{PAA}_{\mathrm{L}} 3.5\right)_{20.5}$} & $10 \mathrm{~s}$ & 2.0 \\
\hline & $1 \mathrm{~min}$ & 3.8 \\
\hline & $5 \mathrm{~min}$ & 5.4 \\
\hline & $10 \mathrm{~min}$ & 9.8 \\
\hline & $15 \mathrm{~min}$ & 9.6 \\
\hline \multirow{5}{*}{$\left(\mathrm{PAH}_{\mathrm{H}} 8.5 / \mathrm{PAA}_{\mathrm{L}} 3.5\right)_{20.5}$} & $10 \mathrm{~s}$ & 2.8 \\
\hline & $1 \mathrm{~min}$ & 3.5 \\
\hline & $5 \mathrm{~min}$ & 3.7 \\
\hline & $10 \mathrm{~min}$ & 5.5 \\
\hline & $15 \mathrm{~min}$ & 5.7 \\
\hline \multirow{5}{*}{$\left(\mathrm{PAH}_{\mathrm{L}} 8.5 / \mathrm{PAA}_{\mathrm{H}} 3.5\right)_{20.5}$} & $10 \mathrm{~s}$ & 19.1 \\
\hline & $1 \mathrm{~min}$ & 99.3 \\
\hline & $5 \mathrm{~min}$ & 128.8 \\
\hline & $10 \mathrm{~min}$ & 140.2 \\
\hline & $15 \mathrm{~min}$ & 158.3 \\
\hline \multirow{5}{*}{$\left(\mathrm{PAH}_{\mathrm{H}} 8.5 / \mathrm{PAA}_{\mathrm{H}} 3.5\right)_{20.5}$} & $10 \mathrm{~s}$ & 20.6 \\
\hline & $1 \mathrm{~min}$ & 119.6 \\
\hline & $5 \mathrm{~min}$ & 141.3 \\
\hline & $10 \mathrm{~min}$ & 187.8 \\
\hline & $15 \mathrm{~min}$ & 222.8 \\
\hline
\end{tabular}

The effect of molecular weight of PAH on the surface topography was not as significant as PAA. When deposited with low molecular weight PAA, the application of high molecular weight 
PAH slightly changed the surface topography by decreasing the size of the islets. For example, when the deposition time was 10s, compared to multilayers built up with the low molecular weight PAH (Figure 3 (a)), the size of the islets for the multilayers with high molecular weight PAH (Figure 4(a)) decreased from $\sim 4$ to $\sim 2 \mu \mathrm{m}$. In addition, as shown in Table 2 , the RMS roughness decreased with the increase of the molecular weight of PAH when the deposition time was longer than $10 \mathrm{~s}$. When deposited with high molecular weight PAA, the application of high molecular weight PAH increased the number of islets on the surface as shown in Figure 5 (b) and (d). Meanwhile, the RMS roughness shown in Table 2 increased by depositing high molecular weight PAH. It is possible that the effect of the molecular weight of PAH on the surface RMS roughness was also influenced by the molecular weight of PAA.
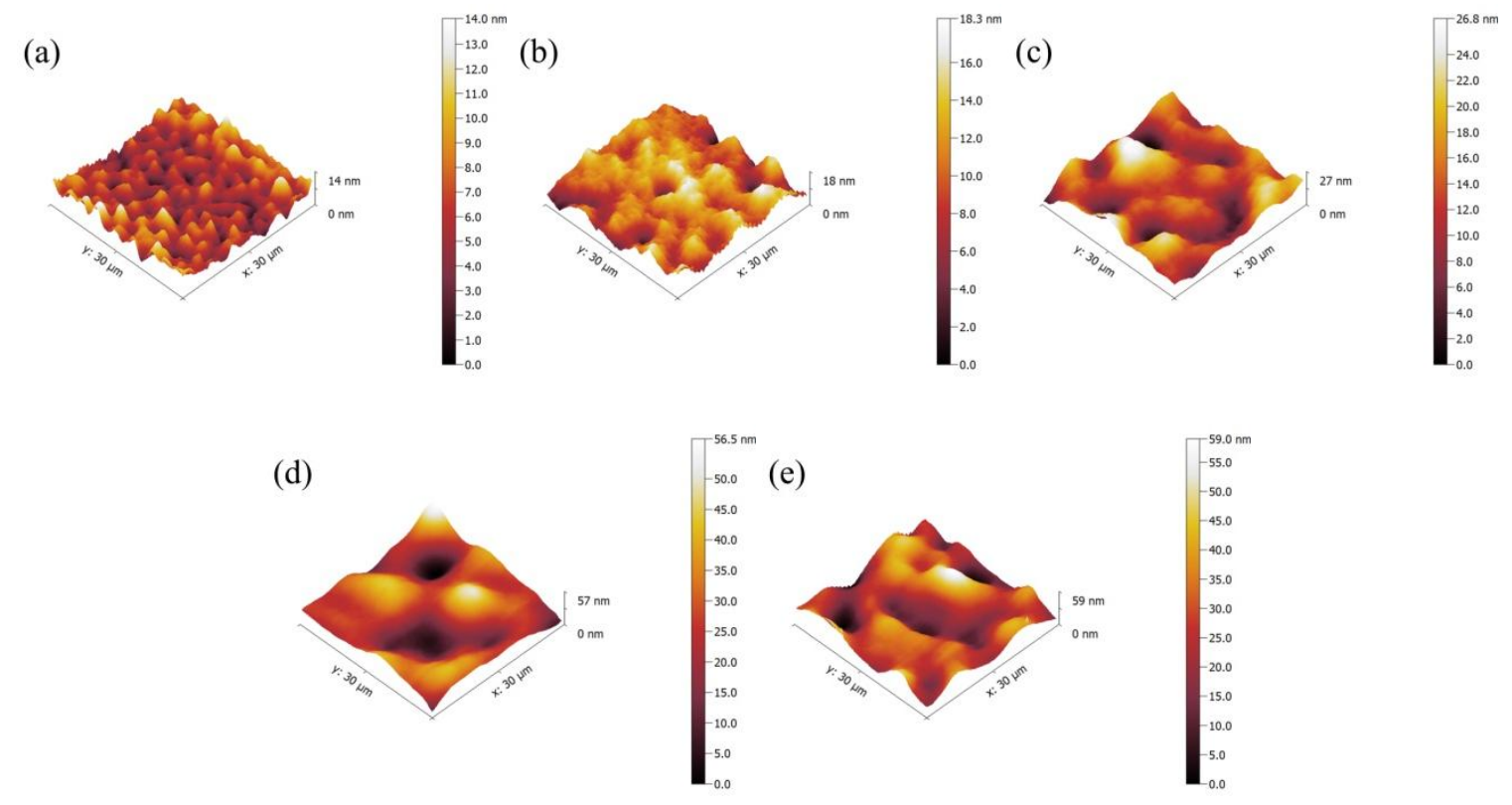

Figure 3. AFM 3D images of the surface morphology of $\left(\mathrm{PAH}_{\mathrm{L}} 8.5 / \mathrm{PAA}_{\mathrm{L}} 3.5\right)_{20.5}$ films with different deposition time: (a) $10 \mathrm{~s}$, (b) $1 \mathrm{~min}$, (c) $5 \mathrm{~min}$, (d) $10 \mathrm{~min}$, and (e) $15 \mathrm{~min}$ 
(a)

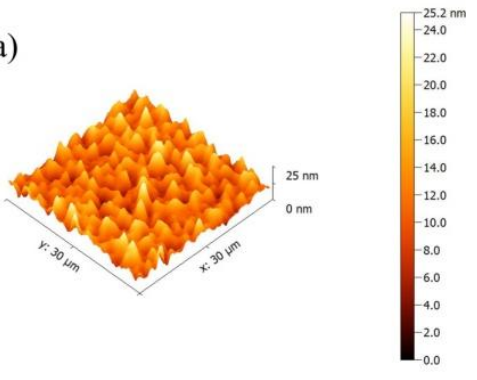

(b)

(d)

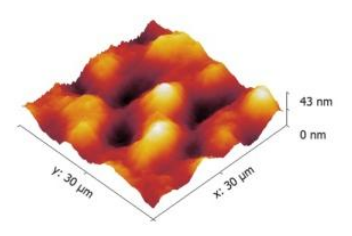

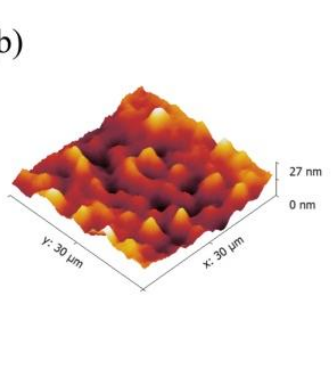

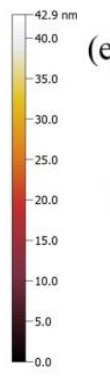

(c)

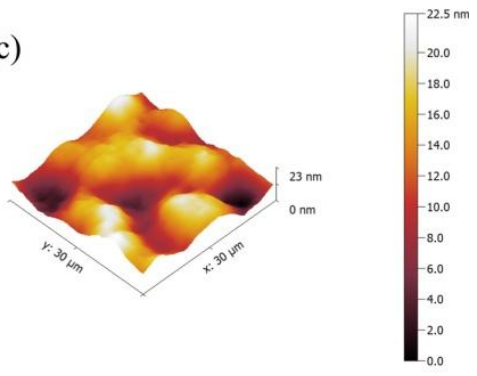

Figure 4. AFM 3D images of the surface morphology of $\left(\mathrm{PAH}_{\mathrm{H}} 8.5 / \mathrm{PAA}_{\mathrm{L}} 3.5\right)_{20.5}$ films with different deposition time: (a) $10 \mathrm{~s}$, (b) $1 \mathrm{~min}$, (c) $5 \mathrm{~min}$, (d) $10 \mathrm{~min}$, and (e) $15 \mathrm{~min}$

(a)

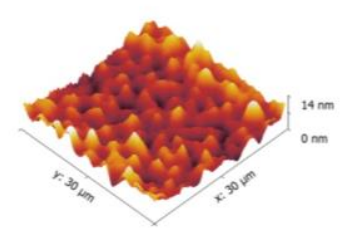

(c)

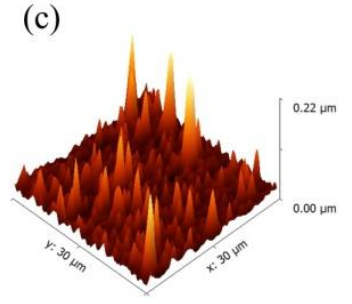

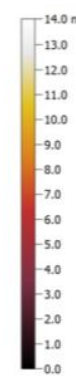

(b)

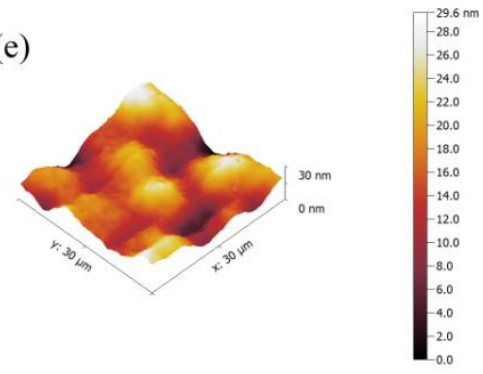

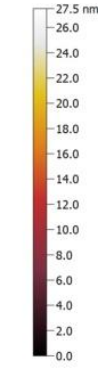

$-29.6 \mathrm{~nm}$
-28.0
-26.0
-2.0
-22.0
-20.0
-1.00
-16.0
-14.0
-12.0
-10.0
-8.0
$6-0$
-4.0
-2.0
0.0 
The Build-up of PAH/PAA Multilayers with Patterned Surface Topography. As shown in Figure 2, when the deposition time increased to $10 \mathrm{~min}$, the deposition of both high molecular weight PAA and PAH formed a unique cantaloupe skin-like structure. It is interesting to study how this unique structure was built up during the LbL assembly. Therefore, Figure 6 presents the surface topography of $\mathrm{PAH}_{\mathrm{H}} 8.5 / \mathrm{PAA}_{\mathrm{H}} 3.5$ system changing with the number of bilayers. The thickness and roughness values are summarized in Figure 7. As shown in Figure 6(a), we found that with the deposition of 4.5 bilayers, the surface consisted of some islet structures. When the number of bilayers increased to 6.5 (Figure 6(b)), some micro-sized circular ring structures were formed on the surface. With a further increase of number of bilayers to 10.5(Figure 6 (c) - (d)), the number as well as the size of the ring structures both increased. Later on, the ring structures started merging together and formed the cantaloupe skin-like structures, as shown in Figure 6 (e) - (i). The thickness and surface roughness values of the multilayers shown in Figure 6 are summarized in Figure 7, accordingly. As shown in Figure 7(a), initially the thickness increased exponentially with the increase of number of bilayers. After the number of bilayers reached 16.5, the increment of thickness slightly decreased. Figure 7 (b) illustrates how the surface roughness changes over the number of bilayers. It is obvious that the RMS roughness always increased with the number of bilayers until the number of bilayers reached 16.5. The RMS roughness maintained almost the same when the number of bilayers increased from 18.5 to 20.5 . The above results indicate that the polyelectrolytes tend to accumulate around rough area on the surface during the initial deposition steps. Therefore, the size of the ring structures and surface RMS roughness increased with the number of bilayer. Once the cantaloupe skin-like structure was formed, the polyelectrolytes started depositing more uniformly on the surface with the surface features maintained almost the same. 

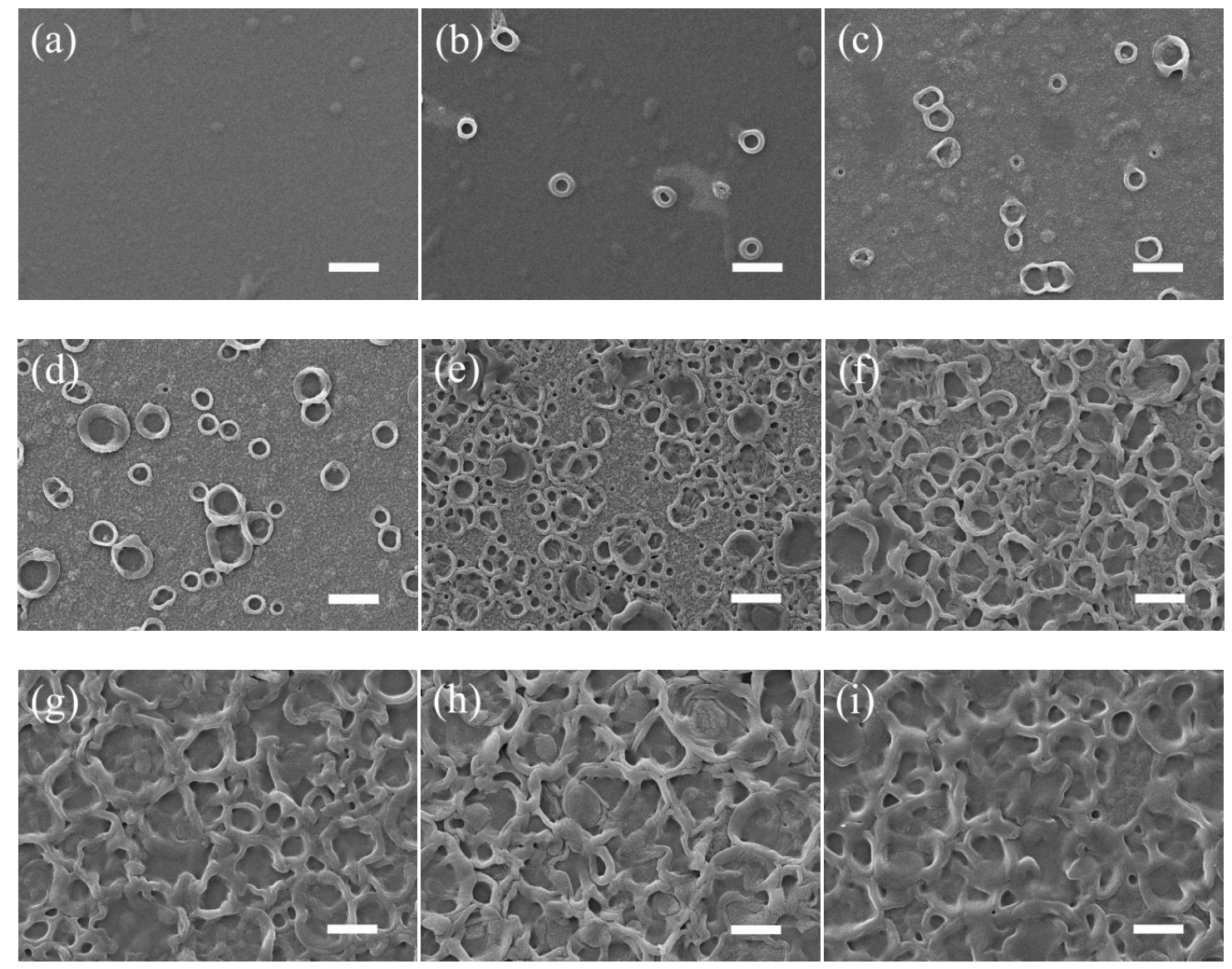

Figure 6. SEM images of $\mathrm{PAH}_{\mathrm{H}} 8.5 / \mathrm{PAA}_{\mathrm{H}} 3.5$ films with number of bilayers as (a) 4.5, (b) 6.5 , (c) 8.5 , (d) 10.5 , (e) 12.5 , (f) 14.5 , (g) 16.5, (h) 18.5 and (i) 20.5, respectively. (The deposition time is $10 \mathrm{~min}$. The scale bar is $5 \mu \mathrm{m}$ for all images.)

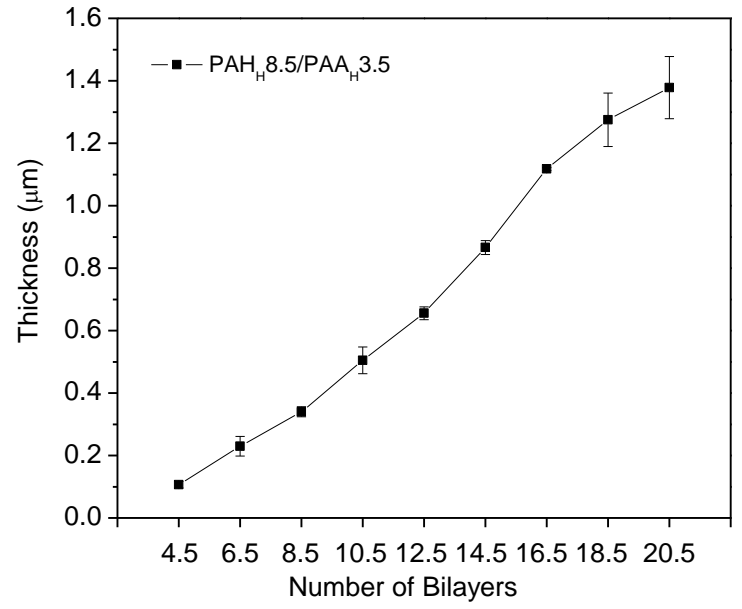

(a)

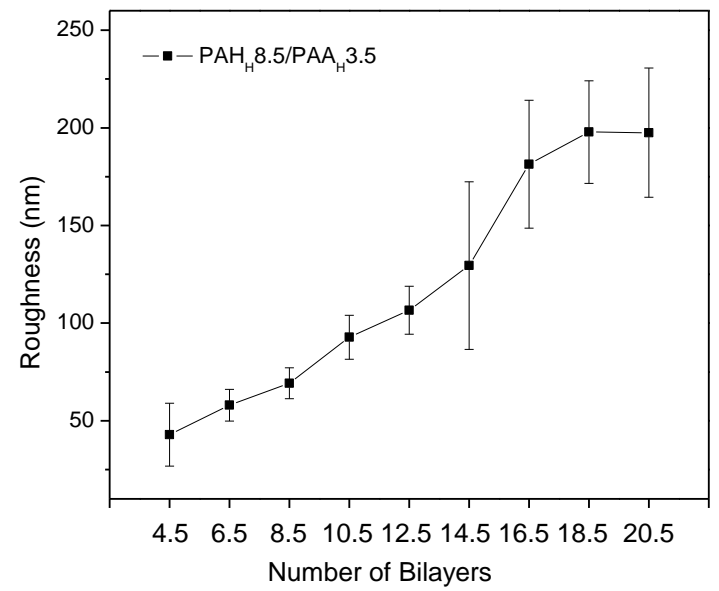

(b)

Figure 7. The values of (a) thickness and (b) roughness of $\mathrm{PAH}_{\mathrm{H}} 8.5 / \mathrm{PAA}_{\mathrm{H}} 3.5$ films as a function of number of bilayers (The deposition time is $10 \mathrm{~min}$.) 


\section{Conclusion}

In this work, we investigated the effect of the deposition time and molecular weight of polyelectrolytes on the film growth and surface topography of PAH/PAA multilayers. The PAH/PAA multilayers were assembled in both exponential and linear growth regimes by adjusting the $\mathrm{pH}$ conditions. In exponential growth regime, the film thickness and surface roughness increased with the increase of deposition time. High molecular weight polyelectrolytes suppressed the interlayer diffusion, leading to the decrease of film thickness. When the deposition time was $10 \mathrm{~s}$ with the interlayer diffusion highly suppressed, the film thickness still decreased by the deposition of high molecular weight PAA, indicating that the adsorption step was slowed down. This was further confirmed by fabricating multilayers in the linear growth regime under the condition of PAH3.5/PAA3.5, where the deposition time may reverse the molecular weight effect on the film thickness. However, the slow adsorption of high molecular weight PAA was only observed when assembled at $\mathrm{pH}=3.5$ with low degree of ionization and poor chain mobility. When PAA was fully charged at $\mathrm{pH}=10$, the film thickness increased with the molecular weight of PAA due to the larger coil size of the high molecular weight PAA. The strong electrostatic force between the molecular chain and the charged multilayer surface facilitated the adsorption step of fully charged high molecular weight PAA. The slow adsorption of high molecular weight PAH was also observed when the multilayers were assembled under the condition of PAH10/PAA10. However, in exponential growth regime, due to the relatively high charge density of $\mathrm{PAH}$ at $\mathrm{pH}=8.5$, no obvious suppression of the adsorption step was found. Therefore, the effect of molecular weight of polyelectrolytes on the film growth is dependent on the degree of ionization and the deposition time. We also found that the deposition of high molecular weight PAA enhanced the surface roughness significantly. Meanwhile, the 
effect of molecular weight of PAH on the surface topography was not as significant as PAA in the exponential growth regime. A cantaloupe skin-like structure was obtained by applying high molecular weight PAA with deposition time longer than $10 \mathrm{~min}$. It was found that the build-up of the cantaloupe skin-like structure started from the structure of small islets and then the formation of circular rings. The unique patterns formed in this work show great potential for the application of cell culture for the sensitivity of cells towards the surface topography.

\section{Acknowledgement}

The fundings from the MSU foundation (Strategic Partnership Grant) and the DOD Strategic Environmental Research and Development Program (DOD SERDP W912HQ-12-C-0020) are greatly appreciated. The authors also would like to thank the W.M Keck Microfabrication Facility at Michigan State University for providing the Dektak Surface Profiler.

\section{References}

[1] G. Decher, Fuzzy nanoassemblies: toward layered polymeric multicomposites, science 277(5330) (1997) 1232-1237.

[2] R. Iler, Multilayers of colloidal particles, Journal of colloid and interface science 21(6) (1966) 569-594.

[3] I. Lee, Molecular Self-Assembly: Smart Design of Surface and Interface via Secondary Molecular Interactions, Langmuir 29(8) (2013) 2476-2489.

[4] Y. Zhu, J. Shi, W. Shen, X. Dong, J. Feng, M. Ruan, Y. Li, Stimuli - responsive controlled drug release from a hollow mesoporous silica sphere/polyelectrolyte multilayer core-shell structure, Angewandte Chemie 117(32) (2005) 5213-5217. 
[5] M.C. Berg, L. Zhai, R.E. Cohen, M.F. Rubner, Controlled drug release from porous polyelectrolyte multilayers, Biomacromolecules 7(1) (2006) 357-364.

[6] H.F. Chuang, R.C. Smith, P.T. Hammond, Polyelectrolyte multilayers for tunable release of antibiotics, Biomacromolecules 9(6) (2008) 1660-1668.

[7] J. Fu, J. Ji, W. Yuan, J. Shen, Construction of anti-adhesive and antibacterial multilayer films via layer-by-layer assembly of heparin and chitosan, Biomaterials 26(33) (2005) 6684-6692.

[8] D. Lee, R.E. Cohen, M.F. Rubner, Antibacterial properties of Ag nanoparticle loaded multilayers and formation of magnetically directed antibacterial microparticles, Langmuir 21(21) (2005) 9651-9659.

[9] M.T. Thompson, M.C. Berg, I.S. Tobias, M.F. Rubner, K.J. Van Vliet, Tuning compliance of nanoscale polyelectrolyte multilayers to modulate cell adhesion, Biomaterials 26(34) (2005) 6836-6845.

[10] A. Schneider, G. Francius, R. Obeid, P. Schwinté, J. Hemmerlé, B. Frisch, P. Schaaf, J.-C. Voegel, B. Senger, C. Picart, Polyelectrolyte multilayers with a tunable Young's modulus: influence of film stiffness on cell adhesion, Langmuir 22(3) (2006) 1193-1200.

[11] M.A. Priolo, D. Gamboa, K.M. Holder, J.C. Grunlan, Super gas barrier of transparent polymer- clay multilayer ultrathin films, Nano letters 10(12) (2010) 4970-4974.

[12] Y.H. Yang, L. Bolling, M.A. Priolo, J.C. Grunlan, Super gas barrier and selectivity of graphene oxide - polymer multilayer thin films, Advanced Materials 25(4) (2013) 503-508.

[13] B. Tieke, F. Van Ackern, L. Krasemann, A. Toutianoush, Ultrathin self-assembled polyelectrolyte multilayer membranes, The European Physical Journal E 5(1) (2001) 29-39.

[14] O. Sanyal, A.N. Sommerfeld, I. Lee, Design of ultrathin nanostructured polyelectrolytebased membranes with high perchlorate rejection and high permeability, Separation and Purification Technology 145 (2015) 113-119.

[15] O. Sanyal, Z. Liu, J. Yu, B.M. Meharg, J.S. Hong, W. Liao, I. Lee, Designing foulingresistant clay-embedded polyelectrolyte multilayer membranes for wastewater effluent treatment, Journal of Membrane Science (2016).

[16] J.A. Hiller, J.D. Mendelsohn, M.F. Rubner, Reversibly erasable nanoporous anti-reflection coatings from polyelectrolyte multilayers, Nature materials 1(1) (2002) 59-63.

[17] Y. Li, S. Chen, M. Wu, J. Sun, Polyelectrolyte multilayers impart healability to highly electrically conductive films, Advanced Materials 24(33) (2012) 4578-4582.

[18] G. Ladam, P. Schaad, J. Voegel, P. Schaaf, G. Decher, F. Cuisinier, In situ determination of the structural properties of initially deposited polyelectrolyte multilayers, Langmuir 16(3) (2000) 1249-1255.

[19] Z. Sui, D. Salloum, J.B. Schlenoff, Effect of molecular weight on the construction of polyelectrolyte multilayers: stripping versus sticking, Langmuir 19(6) (2003) 2491-2495.

[20] A.F. Xie, S. Granick, Local electrostatics within a polyelectrolyte multilayer with embedded weak polyelectrolyte, Macromolecules 35(5) (2002) 1805-1813.

[21] J. Choi, M.F. Rubner, Influence of the degree of ionization on weak polyelectrolyte multilayer assembly, Macromolecules 38(1) (2005) 116-124.

[22] N.S. Zacharia, M. Modestino, P.T. Hammond, Factors influencing the interdiffusion of weak polycations in multilayers, Macromolecules 40(26) (2007) 9523-9528.

[23] S.S. Shiratori, M.F. Rubner, pH-dependent thickness behavior of sequentially adsorbed layers of weak polyelectrolytes, Macromolecules 33(11) (2000) 4213-4219.

[24] P. Bieker, M. Schönhoff, Linear and exponential growth regimes of multilayers of weak polyelectrolytes in dependence on pH, Macromolecules 43(11) (2010) 5052-5059. 
[25] Y.-H. Yang, F.A. Malek, J.C. Grunlan, Influence of deposition time on layer-by-layer growth of clay-based thin films, Industrial \& Engineering Chemistry Research 49(18) (2010) 8501-8509.

[26] F. Xiang, P. Tzeng, J.S. Sawyer, O. Regev, J.C. Grunlan, Improving the gas barrier property of clay-polymer multilayer thin films using shorter deposition times, ACS applied materials \& interfaces 6(9) (2013) 6040-6048.

[27] O. Mermut, C.J. Barrett, Effects of charge density and counterions on the assembly of polyelectrolyte multilayers, The Journal of Physical Chemistry B 107(11) (2003) 2525-2530.

[28] D.A. Hagen, B. Foster, B. Stevens, J.C. Grunlan, Shift-Time Polyelectrolyte Multilayer Assembly: Fast Film Growth and High Gas Barrier with Fewer Layers by Adjusting Deposition Time, ACS Macro Letters 3(7) (2014) 663-666.

[29] S. Micciulla, S. Dodoo, C. Chevigny, A. Laschewsky, R. von Klitzing, Short versus long chain polyelectrolyte multilayers: a direct comparison of self-assembly and structural properties, Physical Chemistry Chemical Physics 16(40) (2014) 21988-21998.

[30] L. Richert, P. Lavalle, E. Payan, X.Z. Shu, G.D. Prestwich, J.-F. Stoltz, P. Schaaf, J.-C. Voegel, C. Picart, Layer by layer buildup of polysaccharide films: physical chemistry and cellular adhesion aspects, Langmuir 20(2) (2004) 448-458.

[31] B. Sun, C.M. Jewell, N.J. Fredin, D.M. Lynn, Assembly of multilayered films using welldefined, end-labeled poly (acrylic acid): influence of molecular weight on exponential growth in a synthetic weak polyelectrolyte system, Langmuir 23(16) (2007) 8452-8459.

[32] P. Kujawa, P. Moraille, J. Sanchez, A. Badia, F.M. Winnik, Effect of molecular weight on the exponential growth and morphology of hyaluronan/chitosan multilayers: A surface plasmon resonance spectroscopy and atomic force microscopy investigation, Journal of the American Chemical Society 127(25) (2005) 9224-9234.

[33] C. Porcel, P. Lavalle, G. Decher, B. Senger, J.-C. Voegel, P. Schaaf, Influence of the polyelectrolyte molecular weight on exponentially growing multilayer films in the linear regime, Langmuir 23(4) (2007) 1898-1904.

[34] M. Paßvogel, P. Nestler, R. Köhler, O. Soltwedel, C.A. Helm, Influence of Binary Polymer Mixtures on the Nonlinear Growth Regimes of Polyelectrolyte Multilayer Films, Macromolecules 49(3) (2016) 935-949.

[35] P. Nestler, M. Paßvogel, C.A. Helm, Influence of Polymer Molecular Weight on the Parabolic and Linear Growth Regime of PDADMAC/PSS Multilayers, Macromolecules 46(14) (2013) 5622-5629.

[36] J. Fu, J. Ji, L. Shen, A. Küller, A. Rosenhahn, J. Shen, M. Grunze, pH-amplified exponential growth multilayers: A facile method to develop hierarchical micro-and nanostructured surfaces, Langmuir 25(2) (2008) 672-675.

[37] D. Yoo, S.S. Shiratori, M.F. Rubner, Controlling bilayer composition and surface wettability of sequentially adsorbed multilayers of weak polyelectrolytes, Macromolecules 31(13) (1998) 4309-4318. 


\section{Graphical Abstract}

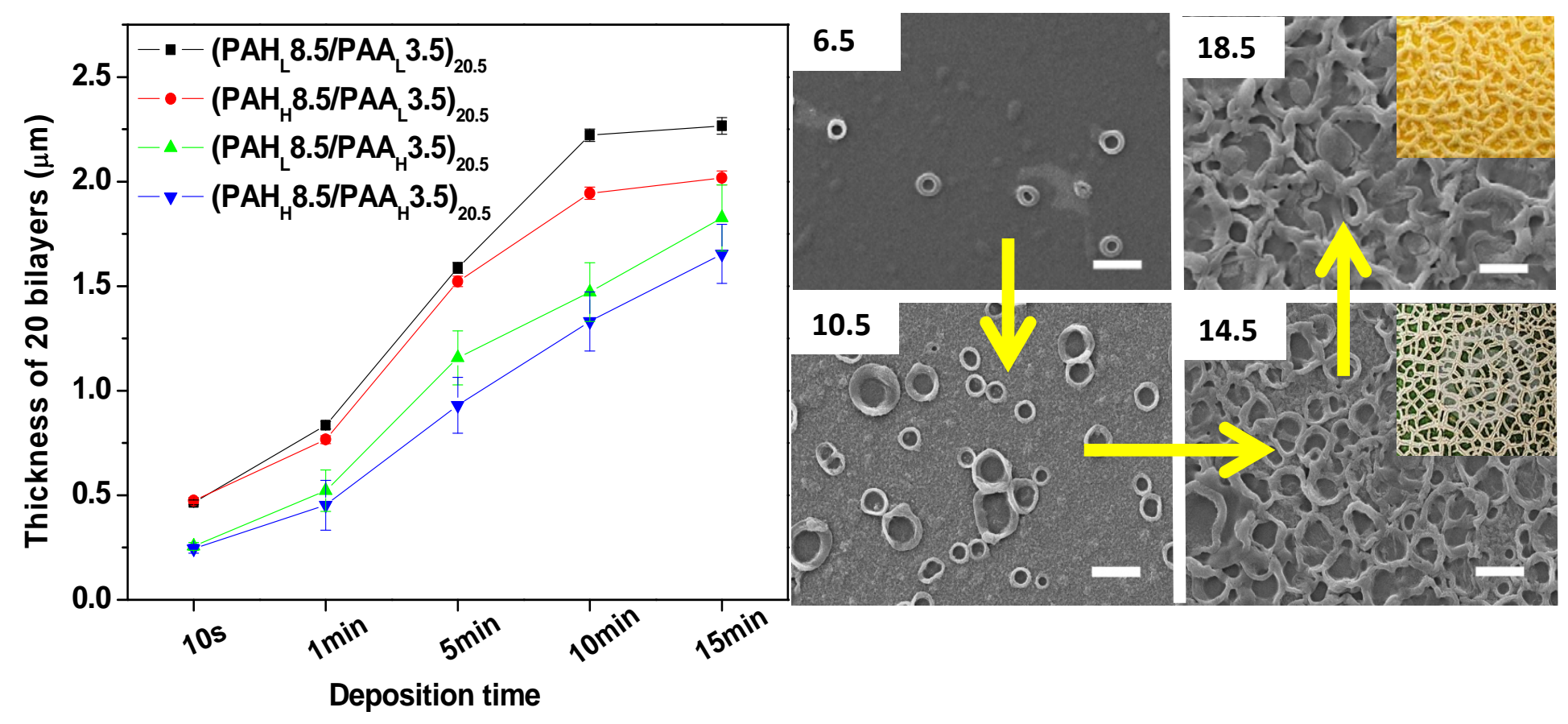

